\title{
Ethical Issues of Psychiatric Nursing in Japan: A Literature Review
}

\section{Ogino $M^{*}$}

Musashino University, Japan

*Corresponding author: Masa Ogino, Musashino University, 1-1-20 shinmachi, nishitokyo-shi, Tokyo 202-8585, Japan, Tel: + 81-3-5530-7333; Email: ma_ogi@musashino-u.ac.jp

\section{Review Article}

Volume 2 Issue 5

Received Date: August 28, 2018

Published Date: September 06, 2018

\section{Abstract}

Aim: The aim of this paper is to examine the latest research and literature related to psychiatric nursing in Japan in order to shed light on the ethical challenges that the field currently faces.

Methods: A literature search was conducted using the keywords psychiatric nursing and nursing ethics within the search system of the Japan Medical Abstracts Society. We examined recent literature (2013-2018) as well as research articles on excited literature.

Result: We examined 24 of literatures related to ethics in psychiatric nursing. These studies showed that psychiatric nurses perceive their ethical dilemmas to be "an inability to respect the patient's wishes," "restricting the movement of patients," "conflict between patients and their families," "interaction with patients," and "insufficient communication between treatment providers."

Discussion: Problems that psychiatric nurses in Japan perceived as ethical dilemmas arise from the nature of mental illnesses and are ethical quandaries that every country must deal with. One of the reasons psychiatric nurses in Japan feel that restricting the movement of patients creates an ethical dilemma has to do with the country's former yet longstanding practice of committing mentally disabled individuals to psychiatric facilities. Some of Japan's cultural aspects that influence ethical issues in psychiatric treatment are its family system and its methods of human interaction.

Conclusion: It is essential that psychiatric nurses understand their own culture and maintain an ethical view as members of the nursing profession.

Keywords: Psychiatric nursing; Mental health; Ethical issues; Ethical dilemmas

\section{Introduction}

As areas such as regenerative medicine and end-of-life medical technology experience innovative growth, our society is becoming increasingly concerned with the ethical issues that arise from medical treatment.

Due to the nature of diseases encountered in psychiatric care facilities, however, the ethical issues that 


\section{Nursing \& Healthcare International Journal}

surround those patients differ from those in other medical fields. Mental illness is unique in that those who suffer from it might not even recognize that they have it, and they may believe that they don't need treatment. But in some cases, these patients are legally compelled to begin treatment if a medical professional determines it to be necessary.

Additionally, psychiatric treatment in Japan involves a different set of ethical challenges than those faced in other nations. WHO [1] surveys have revealed that, compared to other countries, Japan presently has a large number of psychiatric hospital beds and its mental-health patients are admitted to psychiatric hospitals for long periods of time. This paper intends to elucidate the current ethical issues found within psychiatric nursing in Japan.

\section{Purpose}

The aim of this paper is to examine the latest research and literature related to psychiatric nursing in Japan in order to shed light on the ethical challenges that the field currently faces.

\section{History of Psychiatric Practice in Japan}

In the aftermath of the Second World War, Japan adopted a democratic mindset and established laws governing modern psychiatric treatments. But at the same time, the post-war revival of industry led the Public Health Department of what was then the Ministry of Health and Welfare to declare in "Current Situation and Issues of Mental Health in Japan" that "mentally disabled people hinder industry by more than $¥ 100$ billion" and to adopt a policy of committing those individuals to isolated facilities. During this period, the number of mentally disabled people committed to psychiatric hospitals in the United States was also at its peak.

But Kennedy's presidential message to Congress in 1963 set into motion a shift toward welfare policies for mentally disabled people in the US, a movement which eventually ended the practice of shuffling patients off to live in psychiatric facilities. Many other countries which had until then adopted similar policies of committing mental-health patients to psychiatric hospitals also began transitioning to welfare policies that allowed patients to live within their own communities. Japan has lagged significantly behind other advanced nations in this regard, with the Ministry of Health, Labor, and Welfare finally signaling a conversion to community-based policies in the guidelines published in 2006. Still, since many of the country's mental-health patients have already lived in psychiatric hospitals for years, the transition to local care has been slow and unpromising.

\section{Methods}

A literature search was conducted using the keywords psychiatric nursing and nursing ethics within the search system of the Japan Medical Abstracts Society, a computer database of medical literature in Japan. We examined recent literature (2013-2018) as well as research articles on excited literature.

\section{Results}

We surveyed the existing literature and extracted 30 papers, then examined 24 of those related to ethics in psychiatric nursing.

Of the twenty-four papers, ten involved research into the ethical dilemmas faced by nurses engaged in psychiatric care [2-11]; seven involved research into the ethical views and sensitivities of psychiatric nurses [1218]; five were papers discussing methods for solving the ethical issues in psychiatric treatment [19-23]; and two involved research into ethical issues in psychiatric treatment from the viewpoint of the patient $[24,25]$.

Of the research into the ethical dilemmas faced by psychiatric nurses, five were case studies while the other five were investigative studies conducted using questionnaires and other methods. These studies showed that psychiatric nurses perceive their ethical dilemmas to be "an inability to respect the patient's wishes,"'"restricting the movement of patients,"'conflict between patients and their families,"'interaction with patients," and "insufficient communication between treatment providers."

The inability to respect a patient's wishes is an ethical dilemma that occurs when the opinions of the patient and the treatment provider are not in agreement. Due to their symptoms, patients who suffer from mental illness sometimes lose the ability to make rational and realistic decisions. If the wishes of the patient differ from the determination made by the treatment provider in the best interests of the patient, nurses who want to respect the patient's wishes would perceive the situation as an ethical dilemma $[5,6]$.

Restricting the movement of patients involves physically restraining or isolating a patient for the purposes of treatment. Studies that conducted surveys on the ethical concerns of psychiatric nurses suggest that 


\section{Nursing \& Healthcare International Journal}

minimizing such restrictions is one of their biggest worries [4-6]. Some of the studies also presented examples of ethical dilemmas involving restricting a patient's movement to prevent delirium or water intoxication $[9,10]$.

In one example of conflict between patients and their families, the family was opposed to the patient's release from the hospital; in another, an elderly mental-health patient's family did not want to inform the patient that cancer had also been detected. In such cases, nurses who respected the wishes of the family more than those of the patient felt trapped in an ethical dilemma $[8,18]$.

Patient interaction has to do with using the appropriate speech and behavior with patients. The use of coercive behavior or inappropriate speech by nurses toward patients was described as an ethical dilemma. Another point was the need for each treatment provider to reflect on whether his or her own speech and behavior was appropriate in the eyes of the patient $[2,3]$.

Nurses felt that a lack of communication among treatment providers created an ethical dilemma because it made it difficult for the medical team to reach a consensus regarding the treatment plan for a patient and led to the insufficient sharing of information. This is thought to be affected by the hierarchy-based power relationship between doctors and nurses. It is also considered to be a result of the insular nature of psychiatric hospitals in Japan [5,6].

Research focused on clarifying the ethical views and sensitivities of psychiatric nurses has indicated that while such nurses are highly concerned about nursing ethics, they have low levels of awareness about the topic-few have actually read a code of ethics, for example. In addition, the ethical behavior expected of psychiatric nurses included "respecting the human rights of patients (respecting their wishes),"“fulfilling duties and taking responsibility," "protecting the rights of patients," and "caring (showing respect and gratitude to patients)." Both employment position and level of training were suggested as factors that influence this sort of ethical behavior $[12,14,16,17]$.

The research discussing methods for solving the ethical issues in psychiatric treatment noted that ethics conferences and case-study groups are effective ways of resolving such problems, as is improving the ethical sensitivity of nurses [19,21-23]. Workshops were also mentioned as an effective means of increasing the ethical sensitivities of nurses [20].
Research into ethical issues in psychiatric treatment from the patient viewpoint revealed that patients want their nurses to have "good personalities" and to be "respectful to their patients [24,25]."

\section{Discussion}

\section{Ethical Dilemmas Originating from Problems Unique to Psychiatric Treatment}

Problems that psychiatric nurses in Japan perceived as ethical dilemmas included "an inability to respect the patient's wishes" and "restricting the movement of patients." These problems arise from the nature of mental illnesses and are ethical quandaries that every country must deal with.

Psychiatric symptoms are a wholly subjective experience, and it can be difficult to objectively recognize that such symptoms exist. For the patients themselves, the phenomena born from their symptoms constitute their reality, and these symptoms can result in behavior that is harmful to themselves or others. For that reason, many countries have created laws under which individuals can be forced to undergo treatment (without their consent) if a specialist has determined that they require treatment for their mental illness. Japan, too, has enacted legislation that prescribes compulsory treatment in cases where a government-certified physician deems it necessary. Specialists are also legally permitted to restrict the movement of patients to protect their safety.

Treatment providers are operating under the principle of good conduct as they administer treatment and nursing services that are in the best interests of the patient. But there is also an important principle that involves respecting the independence of the patient, i.e. the decisions that patients make for themselves. In psychiatric medicine, it is easy to fall into the trap of paternalism in cases where the patient's symptoms have reduced his or her ability to make autonomous decisions.

\section{Ethical Issues Unique to Japan's Psychiatric Treatment System}

One of the reasons psychiatric nurses in Japan feel that restricting the movement of patients creates an ethical dilemma has to do with the country's former yet longstanding practice of committing mentally disabled individuals to psychiatric facilities. These policies were in effect for so many years that the committed patients have now grown old, and many of them have developed physical illnesses as well. This means that their 


\section{Nursing \& Healthcare International Journal}

movements are being restricted not because of their psychiatric symptoms but to manage their physical diseases. And as we undergo a shift toward psychiatric treatment policies centered on local care, the number of acute medical units at psychiatric hospitals has increased. The rising use of physical restraints on acute patients has become a problem in Japan [26]. In contrast to other nations, Japanese law does not limit the number of days that a patient's movements can be restricted, which has created ethical issues surrounding the employment of such practices over longer periods of time [27].

Furthermore, Japan's abiding policies of committing mentally disabled individuals to institutions has made the country's psychiatric hospitals quite insular in nature. Within such closed environments, the hierarchy-based power relationship between treatment providers became ingrained, and that has been identified as a factor in the lack of communication among those providers $[5,6]$.

Some of Japan's cultural aspects that influence ethical issues in psychiatric treatment are its family system and its methods of human interaction.

Within the unique culture of Japan, human interaction is based on being sympathetic and making the other party feel welcome and at ease; this is achieved through good manners, personal appearance, and addressing the other person using the appropriate speech. The literature reviewed for this paper indicated that nurses perceive the use of coercive behavior or inappropriate speech toward patients to be an ethical dilemma. Psychiatric nursing in Japan therefore tends to place a higher emphasis on behavior and speech that embodies ethical principles rather than the process of internalizing those principles themselves as some sort of mental model. This may be a factor in the declining ethical sensitivities of psychiatric nurses in Japan.

The family system has to do with the form that Japanese families have traditionally taken. Under this system, everything about a family-its property, its name, and its livelihood-is passed from father to son in each generation. It is true that Japanese families are becoming increasingly nuclear in nature and that the number of individuals living alone has risen. Nevertheless, the opinions of families are still given priority over the wishes of the patients themselves. This is yet another ethical issue unique to Japan.

\section{Conclusion}

Ethical issues of psychiatric nursing in Japan originated from problems unique to psychiatric treatment. On the other hands ethical issues caused unique to Japan's psychiatric treatment system and some of Japan's cultural aspects influence ethical issues in psychiatric treatment. Thus, it is essential that psychiatric nurses understand their own culture and maintain an ethical view as members of the nursing profession.

\section{References}

1. WHO (2014) Mental Health Atlas, World health Organization, pp: 69.

2. Inoue $M$ (2017) The ethical viewpoint of nurses in psychiatric hospitals. 59(2): 378-382.

3. Kondo M (2017) The nursing ethics and nursing ethics education recognized by nurses who are practical educator in psychiatric hospital. Nihon Seisinka Kango Gakujyutsusyukaishi 59(2): 373-377.

4. Sugimoto M (2017) The ethical dilemma of nurses who intend to be a psychiatric registered nurse. Nihon Seisinka Kango Gakujyutsusyukaishi 59(2): 245-249.

5. Ankura H (2017) The ethical dilemma and coping to it of nurses of general hospitals, hospitals for children with severe motor and intellectual disabilities and hospitals prescribed by forensic psychiatry. Tyugoku Shikoku chikuKokuritsuByouinKikou KokuritsuRyoyosyoKenkyuGakkaishi 12: 225-228.

6. Konishi R, Usami S, Ohi M, Fukugawa M (2014) Current Ethical Issues and Future Challenges in Psychiatric Nursing: Based on the Pilot Test Outcome. Bulletin of Kumamoto University, School of Health Sciences (Medicine) 10: 37-45.

7. Konuki Y (2017) The ethical dilemmas of nurses in terminal care. Nihon Seisinka Kango Gakujyutsusyukaishi 60(1): 464-465.

8. Sugimoto S (2017) Case report: Ethical issues in informed consent of patients with mental illness and cancer. Kango Jissen no Kagaku 42(5): 11-17.

9. Mori M, Nakamura S, Suyama K (2016) Factors Related to the Decision of Nurses to Use 4-point Bed-railing Restraints for Post-operative Acute Patients With Dementia. Japan academy of gerontologycal nursing 20(2): 57-66. 


\section{Nursing \& Healthcare International Journal}

10. Fukunaga $F$ (2014) The ethical issues of placebo. Nihon Seisinka Kango Gakujyutsusyukaishi 57(2):131-135.

11. Yanagida N (2013) Literature review: nursing to patients with water intoxication. Nihon Seisinka Kango Gakujyutsusyukaishi 56(2): 251-255.

12. Ohnishi K, Kitaoka K, Nakahara J (2016) Relationship between Moral Distress, and Moral Sensitivity on Nursing Practice among Psychiatric Nurses. Journal of japan academy of psychiatric and mental health nursing 25: 12-18.

13. Bando (2015) The relationship of self - efficacy and ethics of nurses in psychiatric hospitals. Nihon Seisinka Kango Gakujyutsusyukaishi. 58(2): 181-185.

14. Nishida M (2014) Nurses' ethical behaviors between patients with mental illness and nurses. Nihon Seisinka Kango Gakujyutsusyukaishi 57(2): 126-130.

15. Bando K (2014) The development of scale in psychiatric nurses' ethical behaviors. Nihon Seisinka Kango Gakujyutsusyukaishi 57(2): 121-125.

16. Yokoyma M (2014) The ethical viewpoint and ethical behaviors of nurses in care to elderly patients. Nihon Seisinka Kango Gakujyutsusyukaishi 57(1): 406-407.

17. Usami S, Tanaka M, Nagai $Y$, Aisawa $K$, Okaya $K$, et al. (2014) Ethical lssues and Coping with Ethical Problems in Psychiatric Nursing. Journal of Japan Academy of Psychiatric and Mental Health Nursing 23(1): 112-131.

18. Tanaka M, Arashi H, Ryu S, Hamada Y, Koyama T (2014) Frequency of Ethical Problems Experienced by Psychiatric Nurses and Their Associated Factors. Tokyo Women's Medical University Journal 9(1): 2129.
19. Murofushi J (2015) The effective conference approach to ethical dilemmas. Nihon Seisinka Kango Gakujyutsusyukaishi 58(1): 234-235.

20. Nakajima F (2015) The structure of ethical viewpoint in psychiatric nurses participated in a workshop. Nihon KenkoIgakkai Zassi 24(1): 17-24.

21. Nakajima K (2017) The trial to raise ethical sensitivity of staffs. Nihon Seisinka Kango Gakujyutsusyukaishi 60(1): 218-219.

22. Kurihara K (2014) The trial of conference in nursing ethics. Nihon Seisinka Kango Gakujyutsusyukaishi 57(1): 240-241.

23. Kanno R (2014) The changes in recognition and behaviors of nurses participated case study meeting. Nihon Kango Gakkai Ronbunsyu Seishin Kango 44: 129-132.

24. Ogino M (2015) Relational ethics in psychiatric nursing: The ethical problems in the psychiatric hospital from the viewpoint of the person with mental illness. The annual bulletin of Musashino University Institute of Nursing 9: 1-7.

25. Ishiyama K (2014) The care to give security to the patient in a seclusion room. Nihon Seisinka Kango Gakujyutsusyukaishi 57(2): 45-49.

26. Noda S (2014) Patient characteristics affected seclusion and restraint in acute unit of psychiatric hospitals in Japan. Psychiatria et neurologiajaponica 116(10): 805-812.

27. Zhang S, Mellsop G, Brink J, Wang X (2015) Involuntary admission and treatment of patients with mental disorder. Neurosci Bull 31(1): 99-112.

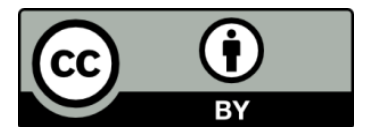

